Criterios y principios para alumnado inmigrante. El Caso de Andalucía

\author{
Fernando Peñafiel Martinez ${ }^{a}$ \\ Rebut: 29/08/2012 Acceptat: 19/12/2012
}

\title{
Resumen
}

A continuación se presenta una de las realidades más acuciantes e inevitables con la que nos encontramos diariamente en las aulas de nuestros centros, la escolarización real y efectiva de alumnos inmigrantes. Los principales retos que afronta desde hace años los centros educativos en España, en general, y más concretamente en los centros educativos andaluces, van dirigidos en dos direcciones: la posible adaptación a la llegada de estos alumnos, que en tan solo una década han experimentado un aumento de más de un $600 \%$, y por supuesto conseguir su máximo éxito dentro del sistema educativo.

En la actualidad, conseguir la integración total de los alumnos inmigrantes constituye un elemento esencial de las políticas educativas, sobre todo si tenemos en cuenta que las escuelas e institutos se han identificado como escenarios claros y marcos privilegiados para que la integración se pueda llevar a cabo en las condiciones más óptimas.

Este trabajo comienza analizando una serie de consideraciones generales en donde se hace referencia a la desventaja sociocultural y las dificultades que conlleva la marginación, así como las necesidades derivadas de una posible organización de la respuesta educativa. A continuación situamos la inmigración en un contexto determinado como es el modelo de sociedad neoliberal, los mecanismos que definen dicho modelo y los valores que la identifican. No olvidamos los planteamientos generales sobre la atención educativa a este colectivo desde los centros escolares

\footnotetext{
a Departamento de Didáctica y Organización Escolar. Universidad de Granada
} 
para finalizar con una propuesta de un diseño de actuación teniendo en cuenta los elementos que la conforman y los objetivos a conseguir.

Palabras Claves: Desventaja social y cultural, marginación, valores, globalización, construcción de la identidad, necesidades educativas, plan de actuación.

\title{
Basic principles and criteria for the design of a plan of action for educational immigrant students. The Case of Andalusia
}

\begin{abstract}
Below is one of the most pressing and unavoidable realities with which we encounter daily in the classrooms of our schools, real and effective schooling for immigrant students. The main challenges facing years schools in Spain, in general, and particularly in schools in Andalusia, are directed in two directions: the possible adaptation to the arrival of these students, who in just a decade have experienced increased over $600 \%$, and of course get your ultimate success in the education system.
\end{abstract}

Today, achieve full integration of immigrant students is an essential element of education policies, especially when you consider that schools and colleges have been identified as privileged frameworks and clear scenarios for integration can be carried out in the most optimal conditions.

This article begins by examining a number of general considerations which refers to the social and cultural disadvantage and the difficulties of exclusion and the needs arising from a possible organization of the education response. Then we put immigration in context such as the neo-liberal model of society, the mechanisms that define the model and values that identify it. Do not forget the general approaches providing education to this group from the school to complete a design proposal for action taking into account the elements that comprise it and the objectives to be achieved.

Keywords: Social and cultural disadvantage, marginalization, values, globalization, identity construction, educational, action plan.

\section{Consideraciones generales}

En la actualidad y en nuestro país, la pertenencia a minorías étnicas y culturales suele añadir problemas a los ya comúnmente conocidos, tales como las dificultades derivadas de la desventaja sociocultural -situación en la que se encuentran las principales minorías existentes en nuestro país-, la pérdida de la propia identidad cultural y personal, los conflictos y cuestiones referidas a los procesos de integración 
y adaptación,... A todas estas, tenemos que añadir otro tipo de dificultades que se derivan de la marginación que se pueden dar en las escuelas, pues se corre el riesgo de no salvaguardar y/o garantizar los valores culturales e históricos de dichos sectores.

Esta posible falta de reconocimiento de los valores con los que el niño se identifica puede llevar, o bien a la infravaloración de su propio grupo cultural, o bien el rechazo de la escuela. Este problema se agudiza en la adolescencia, etapa evolutiva dedicada especialmente a la construcción de la propia identidad, y aún más cuando va unido a la situación de no conocer la lengua de adopción, lo que dificultad el desarrollo de su vida en general y escolar en este caso.

Delgado, Peleg y Luque (2002) tras una investigación realizada, sobre las necesidades educativas del alumnado magrebí en la Educación Primaria en Andalucía, planteaba que la población inmigrante presenta unas necesidades evolutivo-educativas específicas, a tener en cuenta a la hora de desarrollar un plan de atención, y que se agrupan en cinco categorías:

- Necesidades derivadas de su experiencia migratoria, ya que el proceso de llegada y acomodación al nuevo entorno les llena de ansiedad y expectativas. Las necesidades aquí incluidas se refieren a aspectos tales como la seguridad, la atención de las necesidades básicas, los vínculos emocionales y las actitudes hacia la sociedad de acogida.

- $\quad$ El segundo grupo de necesidades vienen dados y derivados por las propias diferencias culturales, cuyo máximo exponente es la lengua, en donde el alumnado inmigrante presentará dificultades de comunicación probablemente por desconocimiento de la lengua de la sociedad de acogida.

- Necesidades derivadas de la condición social: quizá sea este el mayor condicionante de la población inmigrante y por ende del alumnado. Se incluirían aquí las dificultades de acceso a determinados recursos y experiencias.

- Necesidades derivadas de los cambios de la estructura familiar, ya que ésta suele tener variaciones, especialmente en aspectos relacionados con la mujer. La escuela debe ser sensible a los valores familiares no cayendo en paternalismos superfluos y exigiendo la escolarización de todos los menores de 16 años, especialmente de las niñas, y

- Necesidades derivadas de la experiencia escolar. Como ejemplo sirvan las dificultades del desconocimiento de la lengua de acogida. También se presentan problemas de socialización. No debemos olvidarnos que algunos de ellos carecen de una experiencia previa de escolarización o ésta ha sido muy tardía. En el caso de haber estado escolarizados se podrían presentar necesidades ocasionadas por haber recibido un currículo diferente. 
Cualquier plan que pretendamos diseñar para atender educativamente a este sector de la población debe contemplar una serie de necesidades. Así podemos señalar las siguientes:

1. Necesidad del aprendizaje de la lengua de la sociedad de acogida.

2. Atender a distintos referentes culturales; unos procedentes de su entorno escolar y otros resultantes de su entorno familiar.

Si unificamos ambas aportaciones se podría resumir que el alumnado inmigrante presenta una serie de necesidades educativas específicas que se pueden concretar en los siguientes planteamientos:

El primer grupo de necesidades estarán directamente relacionadas con los problemas lingüísticos, ya que el conocimiento de la lengua es el instrumento fundamental para alcanzar la plena integración.

El alumnado inmigrante, en el ámbito de la lengua, presenta una doble necesidad. Por un lado, conocer y dominar el idioma del país al que emigra, como primera medida esencial para evitar el fracaso escolar; por el otro, preservar y consolidar el conocimiento de la lengua materna para una mejor conservación de su identidad cultural originaria. Estas necesidades exigen medidas compensatorias específicas, pues son muchos los casos en que las diferencias entre las dos lenguas afectan también a los caracteres alfabéticos. Además, hay que tener en cuenta las mayores dificultades que presentan los alumnos mayores, que deben integrarse en Educación Secundaria y Adultos, y a los que resulta más complejo la adquisición de la nueva lengua.

Muy relacionados con los aspectos de la lengua están todos los referidos a las culturas originarias de los inmigrantes, así como los problemas que pueden derivarse del choque entre dos enfoques muy diferentes de concebir el mundo, las relaciones sociales, el papel de la mujer en la sociedad, etc. Habría que encontrar el equilibrio entre el respeto a estas culturas y la adecuación a los valores propios de las sociedades democráticas. Esta es una de las tareas más complejas que se intentará abordar desde cualquier conjunto de actuaciones o estrategias que pretendan atender educativa y socialmente a los individuos.

No hay que olvidar, además de la cuestión de la lengua y la cultura, las necesidades socioeconómicas de muchos de los inmigrantes, sobre todo de los procedentes de países del Tercer Mundo. En ocasiones, nos encontramos ante niños con importantes problemas de salud, nutrición, etc., que indudablemente afectan en su rendimiento escolar. También hay que tener en cuenta que el contexto socioeconómico en que se encuentren los alumnos inmigrantes (vivienda, situación laboral de los padres, etc.) va 
a determinar en buena medida su integración en el sistema educativo, y por extensión, en la sociedad.

\section{La inmigración como fenómeno de la sociedad neoliberal}

La sociedad contemporánea tiene algunos elementos comunes que van desde la creciente multiculturalidad, que se hace extensible a todos los aspectos de la vida, hasta el imperativo tecnológico que actúa como irreversible agente de la modernidad, pasando por la ideología neoliberal y sus consecuencias como bien podría ser la globalización, también, en los contextos educativos.

Si bien es cierto que en estos momentos estamos pasando por una grave crisis económica que afecta a toda la sociedad, pero principalmente a la europea, es importante señalar que aún hoy día existe una gran diferencia entre lo que conocemos como primer y tercer mundo, en donde se vive una época de relativa expansión económica, con un influjo importante de dominio de la ideología neoliberal.

La ideología neoliberal se asienta sobre occidente, extendiéndose a sus zonas de influencia como principio ideológico-económico basado en la producción, en el libre mercado y circulación de las mercancías, lo que implica la globalización de los intercambios económicos, de capitales y de personas. Un modelo que galopa y lo absorbe todo fagocitando, inclusive, a la educación, la escuela y el modelo de cultura que en ella se desarrolla (Pérez Gómez, 1999). Se propone un sistema económico basado en la productividad que se basa en la competitividad, la consecución de beneficios económicos inmediatos y la reducción de los gastos en el proceso de producción, además de "sugerir" un impulso del consumo a la vez que genera un mercado todopoderoso con ramificaciones internacionales.

Ahora la inteligencia y la creatividad no está, en exclusividad, en el vértice superior de la pirámide sino que las "posibilidades" se redistribuyen con el objetivo de extraer el máximo rendimiento a las capacidades multitareas que cada sujeto podría llegar a hacer y a la diversificación del mercado.

¿Pero qué hay detrás del término "sociedad neoliberal"?

A mediados de la década de los 70, el modelo desarrollista-consumista, luego de la crisis del petróleo, entra en un cuello de botella, por cuanto se reducen las tasas de ganancias del capital. Y la esencia del capitalismo es aumentar, constantemente, las tasas de ganancias, independientemente de los medios $y$, por supuesto, independientemente de los valores éticos. En última instancia, se inventará una ética, si es que así puede llamarse, subordinada al aumento de las tasas de ganancias, en donde lo más importante es la libre circulación del capital. A tal efecto, para favorecer dicha medida se sostiene: 
- la apertura incontrolada de los mercados,

- la desregulación o eliminación de toda norma para el capital extranjero,

- la privatización de las empresas estatales y de las instituciones que ofrecen servicios sociales (salud, educación, sistemas de jubilación, construcción de viviendas, ...), con la consiguiente reducción del rol del estado y de los gastos sociales,

- lucha prioritaria contra la inflación,

- la flexibilidad en el plano laboral.

Desde este planteamiento, el neoliberalismo requiere de un Estado que asegure las condiciones de estabilidad económica y política, que constituyan un sistema jurídico a fin de favorecer las operaciones del capital transnacional que provean la infraestructura física y que provean la infraestructura humana (recursos humanos) necesaria para la acumulación del capital.

Al respecto, hay que aclarar que los países líderes cumplen con dichas normativas de manera desigual y en tanto convenga a los mismos. De ahí, por ejemplo, las políticas económicas proteccionistas o los subsidios que ejercen, pero a su vez presionan para que los países periféricos no las lleven a cabo.

Por otro lado, con respecto a la globalización (para muchos mundialización) que se deriva de la impronta y sesgo neoliberal en la sociedad actual, es preciso señalar que funciona a modo de impulso teniendo como referente a las nuevas tecnologías y a los medios de comunicación. Es por ello, que la visión que se puede ofrecer sobre la globalización en la sociedad neoliberal y su dimensión en la educación, pasa por reflexionar sobre el propio sentido de ésta. Esta presumible expansión sin fronteras ha generado una radical economía de mercado (de intereses, de concentración de grandes emporios económicos...), donde no existe una idoneidad de mercados, en los cuales no hay igualdad de condiciones y que estas premisas se hacen extensibles, como penosas consecuencias, a la realidad social y educativa.

Posiblemente el llamado "proceso de globalización" se inicia ya en los siglos XIV y XV con el acceso de los europeos a otras tierras, pueblos y civilizaciones que habitaban los rincones hasta entonces desconocidos del planeta. Con la realización de tales viajes, no cabe duda que los pueblos se fueron aproximando y se establecieron los primeros contactos y relaciones que irremediablemente cambiarían para siempre la historia de esos pueblos. Así empezó el más grande e importante ciclo de transformaciones en la historia de la humanidad ya que un numeroso grupo de culturas se cruzaron entre sí.

Desde entonces el mundo no sería el mismo: civilizaciones serían exterminadas, asimiladas o transformadas por este inevitable contacto; pueblos desaparecerían y 
otros serían transplantados a otros lugares lejanos y desconocidos, como los millones de negros arrancados de África, y desplazados por casi toda América como mano de obra en las colonias; nuevas mercancías surgirían para el creciente y cada vez más vigoroso comercio mundial; hábitos de consumo seculares serían transformados y otros nuevos, a su vez, incorporados. De esta forma nuevas culturas sincréticas nacieron y otras se transformaron por ese enriquecedor y a la vez conflictivo contacto. Así Europa se desarrollo notablemente con la riqueza que la "globalización" expansiva le proporcionaba y a través de estas nuevas relaciones también se transferiría para las nuevas colonias muchas de las instituciones europeas, su modo de vida, costumbres, sus religiones cristianas y todo un contenido simbólico y ideológico que marcarían hasta la actualidad el universo cultural de estos pueblos.

Por consiguiente se podría decir que la llegada de los europeos al "nuevo mundo" sería el primer "balbuceo" del proceso de globalización. Es evidente que desde este período las relaciones comerciales entre las diferentes regiones, pese a las dificultades de transporte y comunicación de la época, pasaron a configurar un sistema económico mundial integrado que iría desarrollándose continuamente hasta los nuestros días. Desde ahí también, un flujo continuo de intercambio quedaría establecido y el germen de los futuros estados-nacionales y la formación y transformación de sus instituciones políticas, culturales, sociales, económicas, jurídicas y administrativas quedaron iniciadas.

La Globalización que hoy conocemos tiene un significado más amplio y acentuado, pues es acompañada por los inmensos cambios tecnológicos y la formidable capacidad de transmisión de información que propicia una vía segura para la expansión de los mercados.

El término globalización tiene una conceptuación difusa, como su denotación también es compleja y variable, asumiendo una forma de acuerdo con la perspectiva de quien la interpreta. Se puede entender como un fenómeno relacionado con los profundos cambios sociales y económicos que vienen desde fuera y de cualquier dirección y están fuera del control local. Se asocia actualmente al término numerosas referencias como la facilidad de transmisión de información, el procesamiento y expansión veloz de esta, la estandarización de los hábitos de consumo, la formación de bloques comerciales, la movilidad internacional de los factores productivos y la creciente interdependencia de los agentes económicos internacionales.

Una nueva estructura social dominante, así como una nueva economía global surgen de la interacción de esos procesos y de las relaciones que desencadenan.

En el ambiente académico hay una tentativa por parte de los especialistas en el tema de diferenciarlo de otros términos, como mundialización o internacionalización. Para Roca (1999: 98-99) la globalización es un simple medio por el cual se puede asistir, vía satélite, "al deambular de millones de personas en el corazón de África" o los bombardeos en una guerra distante ("globalización informática"); "por cual unas especulaciones financieras pueden hundir el sistema financiero mundial 
(globalización financiera)"; por lo cual las industrias "pueden cambiar de país buscando mayores ganancias (globalización productiva)"; por el que se puede escuchar la misma música en todo el mundo ("globalización cultural) o "quedamos expuestos a la contaminación causada por Chernóbil (globalización ecológica)".

La mundialización, por el contrario, "es un proceso por el cual se amplía la conciencia de pertenencia al mismo mundo y se crea en un planeta interconectado y interdependiente; es una nueva forma de comprender un espacio que se amplía y el tiempo que se acelera; significa la pertenencia a un mundo único, más humano y habitable, que se experimenta como un único pulso y como un territorio, que por fin llega a ser el hogar del ser humano a través de contactos sociales y mestizajes culturales, del progreso de las comunicaciones y de la integración intercultural." La globalización, en su punto de vista, estaría también preocupada por los "aspectos cualitativos" del ser humano, la cualidad de vida y con un enfoque más cooperativo entre la gente. Como distinción clara entre los dos términos, sentencia: "por la mundialización el mundo nace único, por la globalización económica nace también desigual y antagónico".

La palabra Globalización adquirió, en la interpretación de muchos sociólogos, un significado de fuerza superior simplificadora y homogeneizadora. Según Fernández $(1997,61)$ las fuerzas globalizadoras dan lugar a una intensa multiplicación de los flujos de datos e intercambios y así a nuevas configuraciones de informaciones mundializadas y localizadas "son pasadas por una gigantesca máquina simplificadora que observa tales modificaciones con una extensión incontenible de las fuerzas del mercado, fuerzas que marcharían en una sola dirección que tienden a confundir sus actores económicos y sociales".

Pero hay otra cara de la globalización que presenta otra serie de características menos llamativas, menos "deseables", pero igualmente reales y actuales: la inmigración.

\section{La inmigración en la sociedad neoliberal.}

Estamos viviendo, aunque últimamente con cierto grado de retroceso, una de las olas de inmigración más grandes de la historia de la humanidad. La necesidad de buscar una vida digna, un trabajo del que vivir, una sociedad con libertades y recursos, hace que Europa y América del norte sean las actuales tierras de promisión, los lugares donde todo este ideal es posible. Se huye de las guerras tribales, étnicas o de religión, de la superpoblación, de las prolongadas sequías, de las imparables lluvias o incendios devastadores,... de las hambrunas y epidemias. Se busca, muchas veces, el poder sobrevivir y luego un poco de bienestar socioeconómico, imposible de encontrar donde se ha nacido.

Otros movimientos migratorios son internos de Europa o dentro del mismo estado español. También hay guerras en Europa y situaciones de injusticias y muchísimo 
paro. Se buscan lugares para esperar que acabe una guerra y poder volver, o para mejorar la situación laboral, o buscando el sol y la tranquilidad para vivir los años de jubilación.

El mundo se mueve, las culturas se encuentran, las personas se mezclan. Siempre ha sido así, y la civilización ha consistido en conquistarnos, colonizarnos, invadirnos, pelearnos, aliarnos,... pero siempre se acababa mezclándonos y compartiendo dioses, leyes, abecedarios, numeración, pólvora, brújula, medicina, música, idiomas,...Comprar, vender, intercambiar, compartir, mestizarnos, civilizarnos. Europa después de siglos de colonizar a los demás continentes le toca hacerse la pregunta. Los otros están aquí: ¿les hemos Ilamado o nos devuelven la visita?

Abandonadas las colonias en el forzoso momento histórico de las independencias ya se vino a Europa una primera ola de inmigrantes, fundamentalmente de asimilados culturales. Después empezaron a llegar y continúan llegando un gran grupo que huye de las hambrunas, pestes y faltas de libertad, de economías devastadas, de guerras para hacerse con el poder, de caciques y militares apoyados desde las ex-potencias colonizadoras para seguir sacando las materias primas al mejor precio y venderles los productos manufacturados al peor precio.

Pero como siempre sucede el que está en el escalón de arriba no se acuerda que ha estado en el escalón de abajo. Habrá españoles y españolas que no quieren acordarse de cuando trabajaban en Centroeuropa, en latino América,... o simplemente emigrantes en otras regiones de España.

Antropólogos, historiadores, sociólogos no hacen más que recordarnos que todos somos inmigrantes. Nosotros o nuestros antepasados hemos viajado y cambiado de tierras, países y continentes en busca de algo mejor. En realidad nosotros somos inmigrantes que hemos llegado antes de los que ahora llegan. Una realidad evidente es afirmar que "Todos somos inmigrantes".

\section{Planteamientos generales sobre la atención educativa a este colectivo}

Como hemos señalado al principio de este trabajo, la creciente presencia de minorías étnicas, culturales y religiosas en nuestro país supone un reto para toda la sociedad en conjunto y para el sistema educativo de forma particular. El fenómeno de la afluencia de una población escolar cada vez más heterogénea, procedente de la inmigración, requiere una respuesta adecuada por parte del sistema educativo; aunque, evidentemente, no estamos ante un problema exclusivamente educativo.

Si el papel de la educación es de algún modo destacado, otros sectores del tejido social, como pueden ser las administraciones públicas, han de ofrecer soluciones para una acogida adecuada del que llega, y para una convivencia enriquecedora entre los miembros de una sociedad cada vez más compleja y necesitada de valores 
compartidos. Es por ello que los procesos educativos exigen, para ser exitosos, la contribución de otras instancias de la sociedad.

Entendemos que la sociedad española en su conjunto, y la Comunidad Andaluza no es una excepción, no ha llegado todavía a articular, total y globalmente, una estrategia rotunda y explícita de integración social de la población inmigrante. En la actualidad son muchas las CCAA las que están abordando el desarrollo de planes de intervención adaptados a sus realidades. En Andalucía, se puso en marcha el Plan Integral para la Inmigración en el que participan diferentes Consejerías del Gobierno Andaluz coordinadas desde la Consejería de Gobernación, y que en la que se intenta garantizar los derechos de estos colectivos y responder a sus necesidades y reivindicaciones, desde la igualdad de oportunidades y la equidad.

Con este Plan se pretende responder a la necesaria incorporación a la sociedad de las personas inmigrantes con una concepción multidimensional de los procesos de integración e inclusión, contemplando de forma conjunta y coordinada la actuación de los distintos departamentos de la Junta de Andalucía tratando de aglutinar también los esfuerzos de otras administraciones públicas.

El punto de partida es el de la normalización en la situación social de los inmigrantes, también en la atención educativa, con el horizonte de una plena igualdad de derechos para todos. Se establece también como criterio, el derecho y el respeto a la diferencia, en un marco compartido y bajo el compromiso de construir una sociedad abierta y fundada sobre los derechos fundamentales de toda persona humana.

En el ámbito educativo hemos de plantearnos como finalidad, la de contribuir a crear actitudes favorables hacia una convivencia en paz y en armonía, enseñando a valorar la riqueza que aporta la diversidad. Para ello hemos de señalarnos como objetivo la necesaria preparación para convivir libre, pacífica y solidariamente en una sociedad plural desde el punto de vista étnico, cultural y religioso. Esto requiere en nuestro caso la atención a la diversidad cultural, que conlleva una educación en los valores fundamentales de la dignidad de toda persona humana, de la libertad, del respeto mutuo y de la corresponsabilidad.

Junto a nuestra necesidad de conocer las convicciones, los usos y la mentalidad de los recién llegados, también hemos de hacer ver a las personas que llegan, la necesidad de conocer las convicciones, los usos y la mentalidad de las poblaciones en las cuales piden integrarse, con sensibilidad, sin arrogancias ni prejuicios por ninguna de ambas partes.

Ciertamente, los inmigrantes son portadores de patrimonios culturales dignos de respeto por cuanto de positivo aportan a la humanidad; pero también es cierto que llegan a un ámbito social y humano dotado de una fisonomía cultural y espiritual propia, a la que es preciso también abrirse si queremos hablar de un verdadero encuentro. 
El hecho que se nos muestra es la situación de una pluriculturalidad que, al mismo tiempo que afirma las lógicas diferencias, no debe renunciar a la posibilidad de asumir aspectos enriquecedores de las relaciones interculturales. El punto de partida ha de ser, a nuestro entender, un modelo de escuela abierto a la multiculturalidad, que camine paulatinamente hacia a un modelo de escuela dialógica, posibilitadora de un positivo encuentro de personas y de culturas, que evite caer en antagonismos y en posiciones radicales y que conciba la diversidad cultural como una oportunidad histórica de enriquecimiento.

Ahora bien, no olvidemos que la integración escolar no es un fin en sí mismo, que además haya de imponerse de modo atropellado, sino un medio al servicio de la verdadera integración, que es la integración social, y que pasa necesariamente por una atención a las características personales de cada alumno y alumna, de la manera que sea más adecuada en cada caso. Estamos ante un fenómeno cultural y estructural que nos obliga a llevar hasta sus mejores posibilidades el principio de la atención a la diversidad.

En este sentido la LOGSE planteó y con la LOE se desarrolla, en su concepción pedagógica de mayor novedad, la posibilidad de diversificar el currículo para atender mejor a las necesidades y expectativas de los alumnos. Se propugna un sistema curricular flexible y adaptable a la pluralidad de situaciones que pueden encontrarse en nuestras aulas. $Y$ en lugar de obligar al alumno a adecuarse a un currículo homogéneo, la propia ley introduce una serie de posibilidades de flexibilización que potencian la adecuación del currículo a la realidad concreta del alumnado con el fin de lograr su máximo desarrollo educativo, insistiendo, además, en que la atención educativa se dispense en el momento en que se detecte una necesidad.

Generalmente en todas las Comunidades Autónomas se ha regulado normativamente la escolarización del alumnado extranjero. En Andalucía, la Ley 9/1999 de Solidaridad en la Educación, que asume como objetivo fundamental la efectiva igualdad de oportunidades en la Educación Obligatoria, constituye el marco de referencia para la implantación de una serie de programas y actuaciones de compensación educativa. Estas intervenciones y medidas de compensación se dirigen al alumnado con necesidades educativas especiales, bien por causas asociadas a sus capacidades personales o bien a causas de etiología socio-familiar, pertenencia a minorías culturales.

Se pretende establecer y priorizar las líneas generales de intervención con este alumnado, y que se concreta en las siguientes direcciones:

- La escolarización de los menores inmigrantes con los mismos derechos que el resto de menores de la Comunidad, facilitando el aprendizaje de la lengua de acogida, posibilitando, de esta forma, su integración plena en el ámbito educativo y social. 
- La oferta del Centro Educativo como lugar de encuentro y como espacio favorecedor de la convivencia, el respeto y la tolerancia, posibilitando un clima de intercambio y cooperación entre las culturas y de difusión de los valores democráticos.

- La puesta en funcionamiento de las Aulas Temporales de Adaptación Lingüística (ATALs) que tienen como finalidad desarrollar actuaciones de incorporación e integración del alumnado extranjero, siempre en colaboración con el centro.

Como adelanto de los criterios que son precisos a tener en cuenta se puede señalar que, de partida, hemos de intentar describir las características de la sociedad en la que se inserta el fenómeno de la inmigración, realizando una aproximación al proceso de incorporación de la población inmigrante a nuestro país - profundizando especialmente en nuestra Comunidad Autónoma -, y comprendiendo e interpretando la repercusión social que ello puede desencadenar. Es importante describir y analizar el proceso de escolarización del alumnado inmigrante en los distintos centros educativos - ya sea esa incorporación de forma normalizada o ya iniciado el curso -, ajustando y adecuando los "programas de acogida" que se desarrollan en los centros, describiendo que estrategias son las más adecuadas para atender educativamente a este alumnado: qué métodos se utilizan para el aprendizaje de la lengua de acogida, qué estructuras organizativas se plantean para atender la diversidad, que opinión y actitud tiene el profesorado sobre esta situación y que proponen los centros y la propia Administración como estrategias para abordar una integración educativa favorecedora de otra integración social más global.

\section{Elementos básicos en el diseño general del plan para la atención educativa del alumnado inmigrante en analucia}

El Plan para la Atención Educativa del Alumnado Inmigrante forma parte del I Plan Integral para la Inmigración en Andalucía elaborado y diseñado por la Junta de Andalucía con la finalidad de actuar de forma coordinada desde las diferentes Consejerías de la Junta de Andalucía a través de áreas de intervención: socioeducativa, socio-laboral, socio-sanitaria, recursos sociales, vivienda, socio-cultural, atención jurídica, formación e investigación, sensibilización social y cooperación al desarrollo.

En cada una de las áreas se establecen unos objetivos, unas medidas a desarrollar y unos indicadores de evaluación para valorar la consecución de esos objetivos. Además se incluye un anexo presupuestario que se distribuye por áreas y por ejercicio económico. 
Como es sabido, las competencias en materia educativa, relativas a la enseñanza no universitaria, en Andalucía corresponden actualmente en su totalidad a la Junta de Andalucía, ya desde el 1 de Enero de 1983, cuando entra en vigor el traspaso regulado por el RD 3936/1982 de 29 de Diciembre. Pues bien, la legislación andaluza de referencia en materia de atención a alumnos extranjeros está contenida en la Ley 9/1999 de Solidaridad en la Educación y, sobre todo, en el Plan para la Atención Educativa del Alumnado Inmigrante.

Con la Ley de Solidaridad en la Educación, que asume como objetivo fundamental la efectiva igualdad de oportunidades en la Educación Obligatoria, se constituye el marco de referencia para la implantación de una serie de programas y actuaciones de compensación educativa. Estas intervenciones y medidas de compensación se dirigen al alumnado con necesidades educativas especiales, bien por causas asociadas a sus capacidades personales o bien a causas de etiología socio-familiar, pertenencia a minorías culturales.

En este último apartado se englobarían las intervenciones que se desarrollan con el alumnado extranjero que además podrá ser destinatario, si fuese el caso, de cualesquiera otras medidas vinculadas a necesidades educativas especiales derivadas de otros factores distintos de su condición étnica o cultural, tales como la situación sociocultural desfavorecida, procedencia de familias dedicadas a tareas agrícolas de temporada y/o itinerantes o las capacidades personales.

Así pues, la efectiva igualdad de oportunidades precisa la compensación de las desigualdades de partida - sean de carácter personal, social o cultural -, compensación arbitrada mediante actuaciones previstas en esta ley y desarrolladas en detalle en disposiciones sucesivas. En este marco normativo y conceptual se inscribe la normativa específicamente orientada a la población escolar de origen extranjero.

En este Plan, que supone un nivel de concreción de la Ley 9/1999 de Solidaridad en la Educación, se explicitan siete objetivos que establecen las líneas generales de intervención con el alumnado extranjero, y más específicamente con el alumnado inmigrante:

\section{Objetivo 1}

Facilitar la escolarización de todas las niñas y niños pertenecientes a familias inmigrantes en los mismos términos que el alumnado andaluz.

El Plan expone como objetivo prioritario conseguir la escolarización de todos los niños y niñas inmigrantes, para lo que la Consejería de Educación y Ciencia pondrá en marcha campañas informativas con el fin de divulgar entre este colectivo (con folletos informativos en varios idiomas) en qué consiste el sistema educativo andaluz, sus posibilidades, becas, ayudas o cómo deben actuar durante el proceso de 
escolarización. Asimismo, se insistirá en la importancia de escolarizar a los niños en edad temprana y sobre todo a las niñas, ya que en muchas de las culturas originales de estos inmigrantes no se plantea que las mujeres vayan a la escuela.

Para garantizar una escolarización de calidad, la Consejería de Educación y Ciencia dotará a los centros que acojan a niños inmigrantes de más recursos humanos y materiales, facilitando el acceso a otros servicios complementarios como el transporte, comedores, plazas en residencias escolares o la participación en actividades extraescolares. Además, se contempla el refuerzo de la figura del mediador intercultural que favorezca el acercamiento del alumno y su familia a la institución escolar, los servicios y ayudas de que dispone el sistema educativo.

\section{Medidas:}

- Divulgación entre las familias y colectivos de inmigrantes, de los aspectos básicos del proceso de escolarización y organización del sistema educativo de Andalucía y de las convocatorias de becas y ayudas al estudio.

- Campañas de sensibilización entre la población inmigrante para la escolarización de las niñas y niños en la Etapa de Educación Infantil.

- Dotación de recursos humanos y materiales extraordinarios a los centros que escolaricen a un número significativo de alumnado inmigrante.

- Acceso a los servicios complementarios.

- Acceso a plazas en las Residencias Escolares para que el alumnado inmigrante pueda continuar sus estudios después de cursar las enseñanzas obligatorias.

- Fomento de la participación del alumnado inmigrante en las actividades extraescolares del centro.

- Formalización de convenios con entidades sin ánimo de lucro para establecer la figura mediadora intercultural.

\section{Objetivo 2}

Favorecer que los centros elaboren Proyectos de Centro interculturales que faciliten y promuevan procesos de intercambio, interacción y cooperación entre las culturas.

Los centros garantizarán una educación intercultural a través de un proyecto de centro adaptado, con un currículo integrador, con un plan de orientación y acción tutorial que proporcione al alumno y su familia una información y orientación escolar que favorezca su proceso de integración. Para conseguir este fin se formará al profesorado que va a trabajar con este alumnado y se editarán materiales de apoyo y asesoramiento para los centros y el profesorado. 


\section{Medidas:}

- Formación y asesoramiento específico al profesorado de los centros que atienden al alumnado perteneciente a familias inmigrantes.

- Publicación de materiales de apoyo y asesoramiento para los centros y el profesorado.

\section{Objetivo 3}

\section{Potenciar programas de apoyo para el aprendizaje de la lengua española.}

Todos los inmigrantes deben conocer la lengua española en un plazo corto de tiempo desde su llegada, con objeto de poder integrarse en el ámbito escolar y social.

Para ello, se reforzará el instrumento que ya tiene en funcionamiento la Junta de Andalucía: las aulas temporales de adaptación lingüística (ATAL), más conocidas como "aulas puente".

\section{Medidas}

- Creación de aulas temporales de adaptación lingüística.

- Formalización de convenios con entidades sin ánimo de lucro para apoyar el aprendizaje de la lengua de acogida, con personal cualificado que conozca la lengua propia del alumnado.

\section{Objetivo 4}

\section{Facilitar el aprendizaje de la lengua materna para que el alumnado no pierda su cultura de origen.}

Para ello, se han diseñado distintas actuaciones de apoyo con el fin de organizar actividades en horario no lectivo, elaborar materiales didácticos para la enseñanza de esta lengua materna o traer, mediante convenios, a profesorado de los países de origen.

\section{Medidas:}

- Apoyo a programas para el aprendizaje y desarrollo de la lengua y culturas maternas.

- Elaboración de materiales didácticos para la enseñanza de la lengua materna del alumnado inmigrante. 
- Gestión, ante los países de origen para que faciliten profesorado para que el alumnado hijo de inmigrante tengan un buen dominio de su lengua materna y para que el resto del alumnado del centro pueda aprenderlas.

\section{Objetivo 5}

Favorecer un clima social de convivencia, respeto y tolerancia, en especial en zonas que acogen inmigrantes, fomentando que los centros educativos sean un núcleo de encuentro y difusión de los valores democráticos no sólo de la comunidad educativa sino del propio barrio.

El centro debe ser un núcleo de encuentro y difusión de valores, en el que reine un clima social de convivencia, respeto y tolerancia. Para ello, es importante la participación de los padres y madres inmigrantes en la vida de los centros, así como la integración de los estudiantes en las asociaciones de alumnos.

\section{Medidas:}

- Apoyo a los centros para que pongan en marcha procesos de reflexión y contraste sobre la perspectiva intercultural que impliquen a todos los sectores de la zona en el que se encuentra el centro.

- Impulso de la participación de madres y padres del alumnado inmigrante en los centros docentes.

- Fomento de la participación del alumnado inmigrante en las asociaciones de alumnos y alumnas.

\section{Objetivo 6}

Potenciar el desarrollo de acciones de formación integral de los alumnos y alumnas inmigrantes adultos, interviniendo de forma especial sobre los padres y madres cuyas hijas e hijos estén escolarizados en la educación básica.

La escolarización de los padres y madres inmigrantes, a través de la red de centros de educación de adultos de la Junta de Andalucía, es fundamental para que tengan acceso a la formación básica, mediante planes específicos adaptados a esta población, formando a un profesorado cualificado o colaborando con instituciones que se mueven en este ámbito.

\section{Medidas:}

- Integración de la población inmigrante en la formación básica de adultos. 
- Desarrollo de planes educativos en los Centros de Adultos dirigidos a la población inmigrante

- Formación específica del profesorado de Educación de Personas Adultas que trabajen con población inmigrante.

- Establecimiento de convenios con asociaciones, organizaciones o instituciones que trabajen con la población inmigrante.

- Promoción de la participación de las personas inmigrantes adultas en asociaciones de alumnos, de vecinos, culturales, etc.

- Impulsar Planes de acción Comunitaria que permitan el desarrollo de acciones de integración social.

\section{Objetivo 7}

\section{Impulsar Planes de Integración Social de la población inmigrante más desfavorecida con la participación de las distintas administraciones.}

Todas estas medidas que definen la labor del centro educativo tienen que reforzarse con otras actuaciones en el ámbito sociocomunitario por medio de otras instituciones y administraciones, de manera que se impulsen Planes de integración social de la población inmigrante más desfavorecida.

Estas son las medidas que plantea la Consejería de Educación y Ciencia para atender educativamente al alumnado inmigrante. Nos queda valorar el "cuánto" y el "como" inciden estas actuaciones en la comunidad educativa. Ese es el objetivo de nuestro trabajo.

\section{Conclusión}

Dado el carácter acumulativo que la desventaja supone, para llevar a la práctica los principios compensadores es necesario incluir una educación preventiva que trate de reducir la desigualdad lo antes posible, empezando desde la Educación infantil.

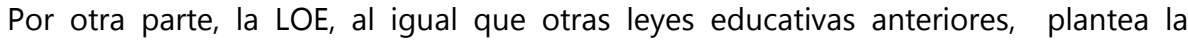
extensión de la escolarización obligatoria con el fin de asegurar la igualdad de oportunidades a todos los alumnos. Esta extensión se convierte en una importante condición de educación compensatoria en función del carácter terminal de la Educación Secundaria. Sin embargo, aunque es una condición necesaria para conseguir que se lleve a la práctica dicho principio, no es suficiente, puesto que exige un diseño curricular que atienda a las necesidades educativas de todos los alumnos. Así mismo contempla también la posibilidad de continuar la formación, mediante programas específicos, de aquellos alumnos que no consigan alcanzar objetivos de la Educación Secundaria Obligatoria. 
La aplicación del principio de igualdad de oportunidades no se logra solamente garantizando la asistencia a la escuela de toda la población en edad de escolaridad obligatoria ni anticipando o ampliando su permanencia en ella, sino que exige además realizar otro tipo de transformaciones cualitativas que permitan eliminar sutiles y complejos procesos de discriminación educativa.

Un análisis minucioso de la desventaja sociocultural y de sus repercusiones en el sistema escolar pone de manifiesto lo incompletos que resultan algunos esfuerzos compensadores, orientados exclusivamente a que sea el niño en desventaja quien se adapte al sistema escolar, sin reconocer la necesidad de transformar al propio sistema.

Entendemos que las situaciones de desventaja que presenta este alumnado a la hora de su incorporación al centro escolar deben de ser abordadas desde una serie de medidas y estrategias para la intervención educativa, fundamentalmente referidos a la metodología y organización, que incidan de forma global en un conjunto de aspectos, que no por desconocidos, deben de ser intencionalmente abordados:

- Un modelo de currículo abierto acompañado de una gran flexibilidad organizativa de los propios centros.

- Adaptación del currículo escolar a las necesidades del alumnado de forma que los aprendizajes sean más significativos y funcionales.

- El desarrollo de programas preventivos para la formación de hábitos y esquemas previos al aprendizaje escolar.

- Reforzamiento de la acción tutorial como elemento favorecedor de la igualdad de oportunidades.

- Desarrollo de la cooperación entre compañeros y compañeras como actitud favorecedora de la solidaridad.

- $\quad$ Propiciar actitudes tolerantes y favorecer las relaciones entre las diferentes culturas originarias del alumnado

- $\quad$ Favorecer y potenciar la relación de la familia con la escuela.

Como podemos observar, todo esto implica desarrollar un marco y una cultura de intervención con alumnado que puedan presentar necesidades específicas asociadas a una situación de desventaja social que, como hemos intentado explicar con anterioridad, correspondería a la mayoría de las situaciones del alumnado inmigrante.

Para profundizar en este tema sería conveniente abordar, de forma más específica, las necesidades educativas específicas del alumnado inmigrante así como las respuestas educativas que normativamente y pedagógicamente se le ofertan y desarrollan en el ámbito de la Consejería de Educación y Ciencia de la Junta de Andalucía. 


\section{Bibliografía}

AA. VV. (1993): Interculturalismo: sociedad y educación. Revista de Educación. Núm. 302. MEC. Madrid

AA. VV. (1994): Interculturalidad y cambio educativo. Hacia comportamientos no discriminatorios. Ed. Narcea. Madrid.

AA.VV. (1991): Minorías étnicas ¿integración o marginación? Cuadernos de Pedagogía, 196. 22

AA.VV. (1997): Educación Intercultural. Cuadernos de Pedagogía. Monográfico diciembre.

AA.VV. (1998) Orientaciones para la escolarización y atención del alumnado inmigrante. CADI. Documentos CPRs Torre Pacheco

AA. VV. (1998), La escuela y sus posibilidades en la formación de actitudes para la convivencia, Apuntes I.E.P.S., Ed. Narcea.

Abad, L.; Cuco, A. E Izquierdo, A. (1993): Inmigración, pluralismo y tolerancia. Edit. Popular. Madrid.

Ala, E. (2000): La inmigración extranjera en España. Fundación la Caixa. Barcelona

Alegret, J. L. (1992): Racismo y Educación. En Fermoso, P. (Ed.): Educación Intercultural. Narcea. Madrid.

Aragón Bombín, R. (1993): Políticas migratorias actuales en España. Polígonos. Revista de Geografía, 3, Pág. 105-134.

Avila Tapies, R. (1993): Nueva perspectiva de las migraciones interiores españolas. Anales de Geografía de la Universidad Complutense, Madrid, n 13, Pág. 111-126.

Blanco Barrios, M. (2001): El alumnado extranjero: un reto educativo. Reflexiones sobre el tema, decisiones organizativas y metodológicas. Editorial EOS, Madrid.

Blanco Barrios, M. (2001): El alumnado extranjero. EOS. Madrid.

Calvo Buezas, T. (1989): Actitudes y prejuicios de los españoles ante los refugiados y ante los extranjeros. In Movimientos humanos en el Mediterráneo Occidental. Barcelona: Instituto Catalán de Estudios Mediterráneos. 259-270.

Carbonell I Paris, F. (1995), Inmigración: diversidad cultural, desigualdad social y educación. MEC. Madrid

CEJA (2001): Plan de Atención Educativa del alumnado inmigrante. Servicios de publicaciones. Junta de Andalucía.

Cohen, A. (1995): Algunas reflexiones a propósito de la inmigración magrebí en España. Ería, 18, p. 287-302. 
Colectivo AMANI (1994), Educación intercultural. Análisis y resolución de conflictos. Edit. Popular. Madrid.

Departamento de Educación Y Cultura (2002): Orientaciones para la escolarización del alumnado inmigrante. Gobierno de Navarra

Essomba, M.A. (Coor.) (1999): Construir la escuela intercultural, Ed. Grao.

Fernández Enguita, M. (1996), Escuela y etnicidad. El caso del pueblo gitano. CIDE, MEC. Madrid

Ferrieri, G. (1996): Aspectos socioeconómicos de las migraciones recientes en España en el contexto de la Europa meridional. Elementos para una posible teoría. Informaciones Geográficas, Alicante, 16, julio-diciembre. Pág. 61-92.

Garcia Roca, J. (1999): Globalización, en 10 palabras clave en filosofía política. Estella.

González Blasco, M. (1999): Aprendizaje intercultural: desarrollo de estrategias en el aula. En Miquel L. y Sans, N. (eds.): Actas de Didáctica del español como lengua extranjera, Madrid, Expolingua, 4.

Guillén Díaz, C. (2004): Los contenidos culturales. En Sánchez Lobato y Santos Gargallo: Vademécum para la formación de profesores, SGEL, Madrid, pp. 835-851.

Jordan, J. A. (1994) La escuela multicultural. Un reto para el profesorado. En Papeles de Pedagogía. Ed. Paidós. Barcelona

Jordan, J.A. (1996), Propuestas de Educación Intercultural para profesores, Ediciones CEAC, Barcelona.

LEY 9/1999, DE 18 DE NOVIEMBRE, de Solidaridad en la Educación. (BOJA 02/12/1999).

LEY ORGÁNICA 2/2006, de 3 de mayo, de Educación (BOE 4/05/2006)

Linares Garriga, J. E (2002): El proyecto educativo de carácter compensador: Reflexiones para su adecuación. Consejería de Educación y Cultura. Región de Murcia

Lluch, X. Y Salinas, J. (1996): La diversidad cultural en la práctica educativa. Ministerio de Educación y Ciencia. Madrid

Miquel López, L. (1999): El choque intercultural: reflexiones y recursos para el trabajo en el aula. En Carabela, 45, Madrid, SGEL, pp. 27-46.

Miquel López, L. (2004): La subcompetencia sociocultural. En Sánchez Lobato y Santos Gargallo: Vademécum para la formación de profesores, SGEL, Madrid, pp. 511- 531.

Monton, M. J. (2003): La integración del alumnado inmigrante en el centro escolar: orientaciones, propuestas y experiencias. Grao. Barcelona 
ORDEN DE 25 DE JULIO DE 2008, por la que se regula la atención a la diversidad del alumnado que cursa la educación básica en los centros docentes públicos de Andalucía (BOJA 22/08/2008).

ORTEGA, R y DEL REY, R. (2004): Construir la convivencia. Edebés. Barcelona.

Peñafiel F., González, D. y Amezcua. J. A. - Coords - (2.003): La intervención psicopedagógica en contextos multiculturales. Granada. GEU

Peñafiel Martínez, F. (1996): Los Valores Educativos en una Sociedad Multicultural. En Ortega, J. A. y Otros: Educación Multicultural para la tolerancia y la paz. Granada. Grupo Editorial Universitario.

Peñafiel Martínez, F. (2005): Logopedia y multiculturalidad. En Peñafiel Martínez, F. y otros: Logopedia para el siglo XXI. Granada. Método.

Peñafiel Martínez, F. y Hernández Fernández, A. (2.006): Respuesta educativa en la multiculturalidad. En Peñafiel, F. y Otros: La intervención en educación especial. Propuestas desde la práctica. CCS. Madrid

Peñafiel, F.; Hernández, A. y Peñafiel, A. (2011): Una perspectiva global de la educación multicultural. Propuestas de intervención desde una perspectiva global. Estudio de caso. En Revista Internacional de Investigación en Ciencias Sociales. Vol. 6 n², pág. 119-136.

Pliego, N. y Valero, M. (2011): Alumnos inmigrantes en España: una realidad creciente. En Hekademos: Revista Educativa Digital. Número 8

REAL DECRETO 1174/1983, de 27 de abril, sobre Educación Compensatoria. (BOE $11 / 05 / 1083)$

Siguan, M. (1998), La escuela y los inmigrantes, Paidós, Barcelona.

\section{WEBGRAFÍA Interesante}

http://www.cadimurcia.net

http://www.pnte.cfnavarra.es/profesorado/recursos/multiculti/el2.php

http://cvc.cervantes.es/obref/inmigracion/

http://www.sgci.mec.es/usa/materiales/

http://www.carmengp.com/caste/

http://babelnet.sbg.ac.at/carlitos/

http://www.ugr.es/ ftsaez/espl2.htm

http://www.um.es/glosasdidacticas/doc-es/primera.html 


\section{Nota biográfica}

Fernando Peñafiel Martinez. Profesor Titular de Universidad de la Universidad de Granada. Profesor del master internacional: "Escritura multilingüe. Procesos cognitivos, interculturales y tecnológicos en la comunicación escrita." Universidad de Granada (España), Universitá Degli Studi di Roma "la Sapienza" (Italia y Anadolu Universitesi, Eskisehir (Turkia). Coordinación Departamento de Didáctica y Organización Escolar de la Universidad de Granada

Facultad de Ciencias de la Educación. Departamento de Didáctica y Organización Escolar. Campus Universitario de Cartuja, s/n. 18071 GRANADA

Despacho 207 (Edificio Central - 10 planta). Telf. +34 958 241343. Fax +34 958 248965. e-mail: penafiel@ugr.es. URL: http://www.fpenafiel.es 